\title{
A systematic approach for performing literature reviews in electrical engineering
}

\author{
Uma abordagem sistemática para revisões da literatura na área de Engenharia Elétrica
}

\author{
G. Bedenik*; J. G. N. Carvalho Filho; E. A. N. Carvalho \\ Electrical Engineering Department/Research Group on Electronic Instrumentation, Federal University of Sergipe, \\ 49100-000, São Cristóvão-SE, Brazil \\ *graziella.bedenik@gmail.com \\ (Recebido em 18 de outubro de 2020; aceito em 26 de abril de 2021)
}

\begin{abstract}
Bibliographic reviews can be limited when researchers face more specific and new challenges. One rising way to solve this is the systematic review. When performing one, there is a well-defined search, treatment, analysis, and display methodology, which follows the scientific method. In this paper, we propose a systematic review methodology for the electrical engineering field. This kind of methodology allows more objective, concrete, and useful results; bias reduction; and easy reproducibility. For clarification purposes, we provide a case study on sensors, transducers, and actuators for controlled environment agriculture, in which the methodology is applied.
\end{abstract}

Keywords: systematic reviews, controlled environment agriculture, electrical engineering.

As revisões bibliográficas podem ser limitantes quando cientistas enfrentam novos e mais específicos desafios de pesquisa. Uma solução em ascensão para tal problemática é a revisão sistemática. Ao realizar uma, há uma metodologia de pesquisa, tratamento, análise e exibição de resultados bem definida, que segue o método científico. Neste artigo, propomos uma metodologia de revisão sistemática para a área de Engenharia Elétrica. Tal tipo de metodologia permite resultados mais objetivos, concretos e úteis; redução de viés; e fácil reprodutibilidade. Para fins de esclarecimento, fornecemos um estudo de caso sobre sensores, transdutores e atuadores para a agricultura de ambiente controlado, no qual a metodologia é aplicada.

Palavras-chave: revisões sistemáticas, agricultura de ambiente controlado, engenharia elétrica.

\section{INTRODUCTION}

We could state science is a cooperative social activity, and knowledge is the result of such interaction. Therefore, literatures reviews are one of the main methods researchers use to organize this knowledge and map initiatives and the state of art in their fields [1].

The most popular "category" for literature reviews is the bibliographic literature review (BLR). They tend to be more descriptive and generic, not requiring rigid systematization of the research process nor its outcomes. The focus is to cover several aspects and studies regarding the topic of interest [2], often without a specific question, on a subarea of the field of study. On the other hand, because they lack a better-defined research methodology, BLRs can be considered partial, limited, and prone to selection bias.

One rising way to solve this is the systematic literature review (SLR), defined by researchers as "the application of scientific strategies that limit bias by the systematic assembly, critical evaluation and synthesis of all relevant studies on a specific topic" [3]. This is because, when performing one, there is a well-defined search, treatment, analysis, and display methodology, following the scientific method. Searches are performed in specified databases using precise search terms, resulting in fewer studies to be analyzed. Also, well-known statistical methods and data extraction tools can be used in these steps, making it easier to draw more concrete conclusions $[2,4]$.

This use of a rigid and well-structured protocol puts SLRs at the top of the hierarchical chain of scientific evidence, which is a "synthesis of better-quality scientific studies on a specific topic or research question" $[1,4]$. This approach emerged in the late 1980s as an investigative methodology supporting the development of Evidence-Based Medicine (EBM). SLRs 
popularization created a demand to standardize the methodology for the acquisition, processing, and analysis of information, to guarantee the reliability of the results and facilitate procedures. This resulted in secondary studies, which are those that gather, summarize, and draw conclusions from the review of primary studies (i.e., sources of knowledge analyzed) $[4,5]$. Although every area of knowledge can benefit from SLRs, not all the details present in the EBM protocol can be applied neither make sense in other fields [6-8].

After EBM became popular, scientists in other health-related areas perceived that, from the use of standardized guidelines and protocols, it is possible to develop and grow study fields based on the accumulation of results from scientific experiments $[1,6,7,9]$. Inspired by this, in the last years, researchers of different areas turned their efforts into developing SLR methodologies for their fields $[8,9]$. It has been successfully used by computer scientists and software engineers "to provide the means by which current best evidence from research can be integrated with practical experience and human values in the decision-making process regarding the development and maintenance of software" [9].

The first engineering SLR-related work is Kitchenham (2004) [6], with the "emergence" of Evidence-Based Software Engineering (EBSE). In Kitchenham (2004) [6] and Kitchenham and Charters (2007) [7], the authors list and discuss their guidelines for performing SLRs in software engineering. The methodology comes down to three phases: Planning, Conducting, and Reporting, based on those established in the health and human sciences' SLRs. Although the methodology is rigid, with a pre-established protocol, the authors stress the caveat that the completion of the steps is not strictly sequential, but rather iterative and interactive. This means search parameters could be modified or refined to obtain more coherent results.

Based on Kitchenham's guidelines, Biolchini et al. (2005) [1], present their work. Their goal is to simplify and detail the concepts and developments related to the systematic review and its guiding question. The three-phase structure is maintained, but they are now called Planning, Execution, and Result Analysis. In these phases, they inserted stages for evaluation and conflict resolution between reviewers. Also, the division of stages, in general, is better established. One of the main contributions of their work is the focus on helping researchers unfamiliar with the practice of SLRs, just as we intend to do with electrical engineering.

Despite these efforts, it is noticeable there is a gap between other engineering areas and the practice of systematic reviews, creating a lot of space for growth and usage of the SLRs benefits. As an example of this potential, so far, no articles or projects have been found directly involving electrical engineering and SLRs.

It is based on the protocol for SLRs in software engineering proposed in Biolchini et al. (2005) [1]. This kind of methodology allows 1) more objective, concrete, and useful results, due to the quality of being an evidence-based process, seeking to answer a guiding question; 2) bias reduction, due to the method reliability, with pre-defined criteria for selection and disposal of references; 3 ) easy reproducibility, due to well-determined and disclosed steps [1,6].

Because of these advantages, it is already customary in several areas, when facing a more specific research challenge, to conduct a SLR. One example of such challenges can be that, recently, the continued population growth and the scarcity of natural resources have been worrying governments and institutions about food security for this and the next generations [10, 11]. The use of science and technology in agriculture represents a solution with the potential to increase quantity and quality of food production worldwide. In this sense, we apply the proposed methodology in a SLR on sensors, transducers, and actuators for controlled environment agriculture (CEA), focusing on small growth chambers for research.

Growth chambers configure one of the best ways to implement CEA in academic and even domestic environments [11, 12]. Detailed and complete sensing within the chambers is a fundamental step, since different projects and crops have different demands for growth conditions, and maintenance [13]. However, the problem in the use of sensor elements and actuators falls in the selection and effective use of them, which is not so simple. This is due to both the different characteristics of the elements (scale, range, resolution, precision, accuracy, durability, and price), as well as to solve the particularities of each plant culture, also taking into consideration the purpose of cultivating them [11]. Performing a SLR on the matter is an 
evidence-based approach to finding out what techniques and elements researchers already tried and validated in this research field, improving and supporting technology adoption decisions [14].

More importantly, considering the shortage of SLRs in the engineering field, by carrying out this case study following the proposed methodology, we seek to introduce the practice of systematic reviews in electrical engineering and its potential on information organization, treatment, and analysis.

\section{MATERIALS AND METHODS}

The general idea of the SLR methodology is to ask a guiding question regarding a topic of interest and, at the end of the review, answer the question and achieve your outcome measures [2]. More generally, this process can be seen as a three-step approach [1], as illustrated in Figure 1.

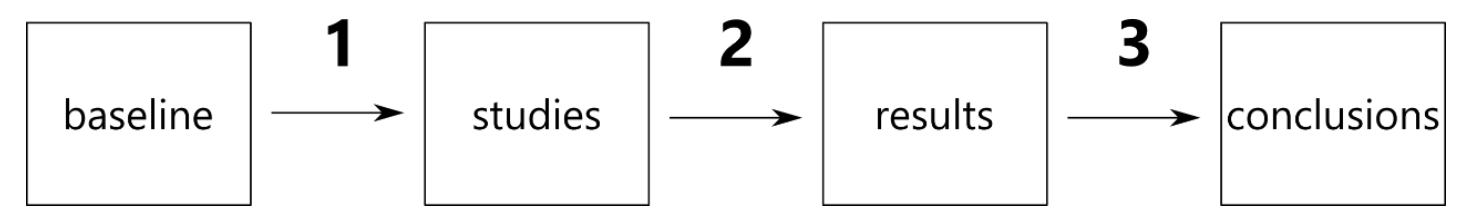

Figure 1: Three-step approach to developing a systematic review of literature. Adapted from Biolchini et al. (2005) [1].

To make this possible, it is necessary to plan what parameters are relevant when searching for studies, as well as strategies to select and analyze them. This prior organization will result in an objective, evidence-based, reliable, and reproducible research protocol.

Although there are already well-defined SLR protocols for health sciences, these cannot be readily used for engineering. For example, some relevant issues concerning SLR use in engineering areas, are 1) the level of evidence produced by clinical trials cannot be directly compared to that produced in most engineering academic experiments; 2) fewer primary studies are performed in engineering, restricting search parameters and sources; 3) double-blinded experiments are generally impossible for engineering experiments [6, 7]. These characteristics result in engineering SLR's being simpler and needing fewer parameters.

In this context, we propose a methodology for performing SLRs in electrical engineering that is divided into three phases: Planning, Execution, and Result Analysis. Each phase has its stages and procedures to allow further detailing and facilitate the following of the protocol.

\subsection{Planning}

In the Planning phase, researchers must outline the goals and the approach to be performed. We will now detail the stages regarding the planning development.

\subsubsection{Question formulation}

This stage is divided into two fundamental procedures to obtain the greatest possible clarity in defining the SLR goals. These procedures are:

a) Question Focus - define the search goals, i.e., what you want to answer at the end of the review.

b) Question Quality and Amplitude - transform goals into the guiding question. This is achieved by defining the "question syntax", which uses the following parameters:

- Problem - define the SLR target, briefly contextualizing it; 
- Auxiliary Questions - define smaller issues that help to build the guiding question;

- Keywords and Synonyms - define the main terms related to the purpose of the review, which should be present in the question(s);

- Intervention - define what will be observed in the context of the review;

- Effect - define the type of results expected after conducting the review;

- Outcome Measure - define the metrics used to measure the effect;

- Population - define the "group" that will be observed in the review;

- Application - define functions, professions or areas that will benefit from the results of the review.

\subsubsection{Sources selection}

This stage is divided into five fundamental procedures for planning how the search for studies will be conducted. These procedures are:

a) Sources Selection Criteria Definition - define which characteristics are necessary for the reference to be a candidate for the SLR.

b) Studies Language - define the languages in which research will be performed. This choice can restrict or expand databases options.

c) Studies Identification - define the strategy to search for initial studies, which uses the following parameters:

- Sources Search Method - description of how to search for references (manually, using search tools online, in libraries, etc.);

- Search String - definition of keywords used in online search engines. Expressions should use logical operators (AND, OR, NOT, truncation, etc.) to obtain as many relevant results as possible;

- Sources List - a preliminary list of databases that can be used.

d) Sources Selection After Evaluation - determine the definitive list of databases, connecting the preliminary list and the Database Selection Criteria.

e) References Checking - define the process of reviewing the source list. It could be performed by one or more experts in the area, and they may add or remove items if necessary.

\subsubsection{Studies selection}

This stage is divided into three fundamental procedures for describing the approach to select references. These procedures are:

a) Studies Inclusion and Exclusion Criteria Definition - define conditions for including or excluding studies, which can be found in the literature or can come from the authors. The criteria should assist in selecting the most effective compilation of studies for the review.

b) Studies Type Definition - this relates to the type of primary studies to be sought, such as qualitative, quantitative, case studies, characterizations, etc.

c) Procedures for Studies Selection - describe the steps for obtaining and evaluating studies, taking the criteria into account.

Although it is not our goal in this paper to discuss or exemplify it, we would like to point out that there are, among studies selection strategies for engineering reviews, mainly in software engineering, an inclination for using search automation tools and methods. They emerge in the context where global scientific production increases at an exponential rate, while SLRs are presented as aggregators and analyzers of the available evidence [4]. 
These mechanisms facilitate the reproduction of reviews and the comparison and reduction of results bias, also contributing to the strengthening of practice in the field of engineering. This is because the practice ends up even contributing to the study and development of systematic search algorithms, which can be an interesting problem for electrical engineering and its ramifications [9].

\subsubsection{Planning evaluation}

The most common evaluation method for Planning Evaluation is to ask experts in the field to run a reduced version of the SLR based on the planned protocol, and then observe development and achieved results.

\subsection{Execution}

In the Execution phase, the search for studies, according to the established criteria, is started and the relevant information and results are extracted from these studies. We will now detail the stages regarding the execution development.

\subsubsection{Search engines evaluation}

Evaluates the engines to be used. This is done through tests with the search string, analyzing search outputs. Depending on this result, if necessary, one or more search engines can be eliminated from the protocol or the string can be modified.

\subsubsection{Selection execution}

This stage is divided into three fundamental procedures to obtain and document the studies according to the criteria and parameters defined. These procedures are:

a) Initial Studies Selection - perform the planning protocol to obtain the initial studies;

b) Studies Quality Evaluation - submit references to all of the defined assessment criteria and record the results;

c) Selection Review - check selected references to ensure that no relevant study has been discarded and record the results to define a final list of studies.

\subsubsection{Information extraction}

This stage is divided into four fundamental procedures for extracting relevant information from the selected studies. These procedures are:

a) Information Inclusion and Exclusion Criteria Definition - define the criteria by which information will be judged as relevant or not.

b) Data Extraction Documentation - define structures and tools to standardize documentation and representation of the extracted information.

c) Extraction Execution - this procedure is divided into two parts:

- Objective Results Extraction - objective results are those directly extracted from the references, which need the following parameters:

Study Identification - study ID, title, authors' names, publishing media information, database, and year of publication;

$>$ Study Methodology - methods used in the study; 
Study Results - effects obtained by the study;

$>$ Study Problems - limitations listed by the author of the study.

- Subjective Results Extraction - subjective results are those not directly extracted from the references, which need the following parameters:

Information Through Authors - to clarify doubts or details;

$>$ General Impressions and Abstractions - conclusions drawn by the reviewers, combined with previous knowledge.

d) Resolution of Divergences Among Reviewers - establish, in case of divergences, a discussion among the reviewers in relation to the acquired information.

\subsection{Result analysis}

In the Result Analysis phase, the results are summarized to communicate information extracted from previous stages in an easy-to-understand and objective form. Qualitative and quantitative analysis may also apply if described in the planning. Relevant information should be displayed in tables and, if coherent with the results, also in graphs and figures. The reviewers should also do an analysis regarding search, selection, and extraction bias. This means describing information found or perceived in any phase of the review that might invalidate the results. Inclinations on the field of study to publish only positive results and particular researchers' viewpoints towards the subject can also be included, representing the publication bias [1].

To conclude the SLR, the authors make the final comments. They discuss and interpret results related to the protocol, as well as the systematic review goals. They may mention the number of studies throughout the review and discuss possible conflicts of interest, inclinations or trends that could have influenced the results. Lastly, the reviewers define possible fields and demands in which the results could be applied, giving suggestions on how the results could be used in the listed applications.

A visual summary of the previously described proposed methodology can be seen in the flowchart in Figure 2, emphasizing phases and their stages. It is interesting to notice the iterative and interactive character of the protocol, evidenced by the process of refinement of it and already demonstrated in methodologies for other areas $[1,6]$.

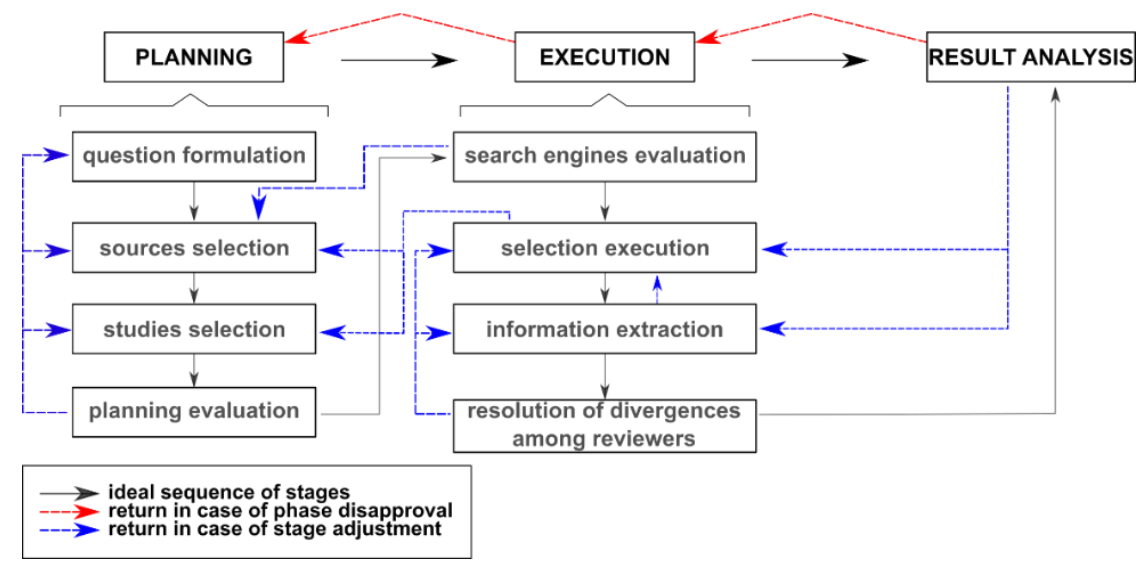

Figure 2: The process of performing a systematic literature review according to the proposed methodology.

\section{RESULTS AND DISCUSSION}

In this simplified example of a systematic review, the focus is on identifying and listing which sensors, transducers, and actuators scientists are currently using in the construction of small 
growth chambers. This culminates in our guiding question, "what sensing and actuating elements have been used in small growth chambers in the last ten years?".

To further develop our protocol, it is necessary to start by establishing the planning parameters. Our problem is that different cultures have different physiological needs. The characteristics of the environments in which we insert these cultures must be adequate for the plant to develop properly. Controlling an environment for this purpose depends on an adequate data acquisition system. When searching for targeted studies, our keywords and synonyms were: controlled environment agriculture; system; instrumentation (measurement, sensing, monitoring, automation).

The intervention concerns the cultures scientists use to test growth chambers and, of course, which sensors, transducers, and actuators they use. The expected SLR effect is a list of potential instrumentation tools to use in the construction of a small chamber for research purposes. The outcome measure is that, in this list, there are at least sensing or actuating elements for all our relevant variables (air temperature, air humidity, soil moisture, and luminosity). The case study population regards CEA systems in general. In the short term, research involving the sensing of agricultural systems and the researchers involved in it are the main application interests. We selected our sources (IEEE Xplore, Springer Link, and ScienceDirect, which we recommend) based on the possibility of online query and credibility and relevance in the field of electrical engineering.

To start the search process, English was the chosen language, and the base search string was: "controlled environment agriculture" AND (small system OR household OR domestic) AND (measure OR instrumentation OR monitoring OR sensing OR automation).

The search string needed modifications for each search engine, which were used in the advanced search option. When possible, filters for a period (2010-2020) and area of study (engineering) were also applied, but we did not restrict the type of studies. After that, for an initial selection, we read the title of each study. Subsequently, the initial studies were completely read and, after a new analysis according to the established criteria, we had an intermediate list. These criteria were that the texts must mention sensors, transducers, and actuators used in small CEA systems. References older than 2010 or that did not mention these elements, or that are related to medium or large systems were not considered. For these selected studies, we also checked their references following the same procedure to search for more studies and then make the final list. Commercially available systems were not examined.

We considered the model's name, brand, and specifications of sensing and actuating elements as relevant information, as well as the culture used for tests. Control techniques and other agrarian aspects were not registered. We used Zotero and Google Sheets to document and manage studies and their information.

By inserting the search string on the selected sources, we first found two hundred thirty-eight studies. After the initial selection, fifty-four of them ended up on our list. After selection criteria analysis, eight studies were pick, and there were no divergences among the reviewers. In this paper, as the review itself is not the focus of the work, but rather an example on how to apply it and a way to facilitate the understanding of it, we will show the procedures performed for only one of the selected studies. We encourage the reader to access the spreadsheet with the complete data on the link https://docs.google.com/spreadsheets/d/1zFwrobRkh4_W3Tsqh_sCYr2y6v5MUb-bsC6gswH1Gc/edit?usp=sharing .

- Identification: ID \#3; "Personal food computer: A new device for controlled-environment agriculture"; E. C. Ferrer, J. Rye, G. Brander, T. Savas, D. Chambers, H. England, and C. Harper; Future Technologies Conference, pp. 1077-1096; Springer; 2018.

- Summary Methodology: the PFC is an open-source open-hardware platform that prioritizes desktop size, low cost, customizability, user-friendliness, and open information. The sensors, actuators, and interfaces of the PFC represent a wide range of devices one can find in several engineering sub-domains.

- Summary Results: for its size, price, and capabilities, it is a complex system that can be used not only as a research platform but also as an educational tool. The open-source 
nature of the PFC improves the quality of the support for the end-users by providing full access to knowledge at every level.

- Problems: not described by the authors.

In Table 1, we can see a reduced version (for study \#3 only) of the list of sensors, transducers, and actuators used in the selected studies for each quantity. This list was the main goal of this sample SLR, and we can see that there are sensing or actuating options for various variables (air temperature, air humidity, soil moisture, and luminosity) even within the reduced version.

Table 1: Sensors, Transducers (S/T) and Actuators (A) for Study \#3.

\begin{tabular}{ccc}
\hline Type & Quantity & Name/Model/Brand \\
\hline S/T & Temperature and air & AM2315 \\
$\mathbf{A}$ & humidity & Thermostatic PTCheating element \\
$\mathbf{A}$ & Temperature & KippKitts cooling unit \\
$\mathbf{A}$ & Air humidity & Phtronics bottle cap air humidifier \\
$\mathbf{A}$ & Soil moisture & Homecube peristalticliquid pumps \\
$\mathbf{S} / \mathbf{T}$ & Luminosity & Adafruit TSL2561 \\
$\mathbf{A}$ & Luminosity & GE light modules \\
$\mathbf{S} / \mathbf{T}$ & pH & Atlas pH sensor \\
$\mathbf{S} / \mathbf{T}$ & Water level & LLE102000 \\
$\mathbf{A}$ & Airflow & DC blower (generic) \\
$\mathbf{A}$ & Airflow & Motorized ballvalve (generic) \\
$\mathbf{S} / \mathbf{T}$ & Electric conductivity & Atlas EC \\
$\mathbf{S} / \mathbf{T}$ & Image & ELP 5 MegapixelUSB camera \\
$\mathbf{S} / \mathbf{T}$ & $\mathrm{CO}_{2}$ concentration & MHZ16 \\
\hline
\end{tabular}

In Figure 3, we can see the number of selected studies that mention instrumentation tools for each quantity, evidencing the relevance of air temperature, air humidity, air flow, soil moisture, luminosity, and soil $\mathrm{pH}$. "Other" refers to water level, irradiation, pressure, water temperature, image, electric conductivity, water oxygenation, or carbon dioxide concentration.

Lastly, in Figure 4, we can see what cultures the authors of the selected studies used to test their growth chambers. Tomato was the most common one, but curiously, it is also the most challenging one, due to its sensitivity to diseases and environment variations [12].

Since this sample review is set only as an example for those interested, we did not focus on defining search, selection, and extraction bias nor publication bias, although we can say right away that we worked with only a few databases and did not use search automation tools, as the one presented in Zumaeta et al. (2019) [15], for performing the Execution phase. 


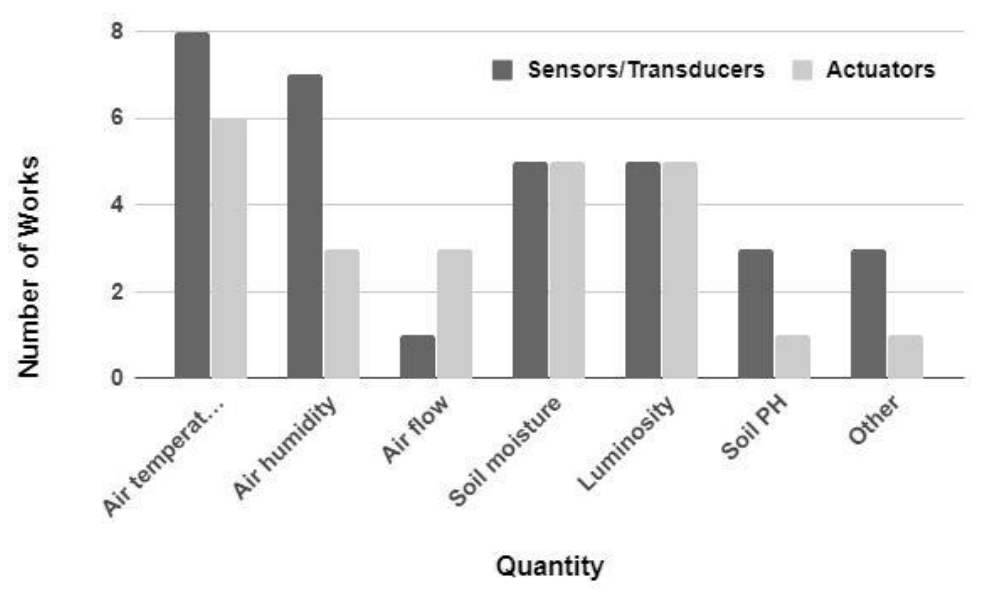

Figure 3: Number of selected studies that use sensors, transducers, and actuators for each quantity.

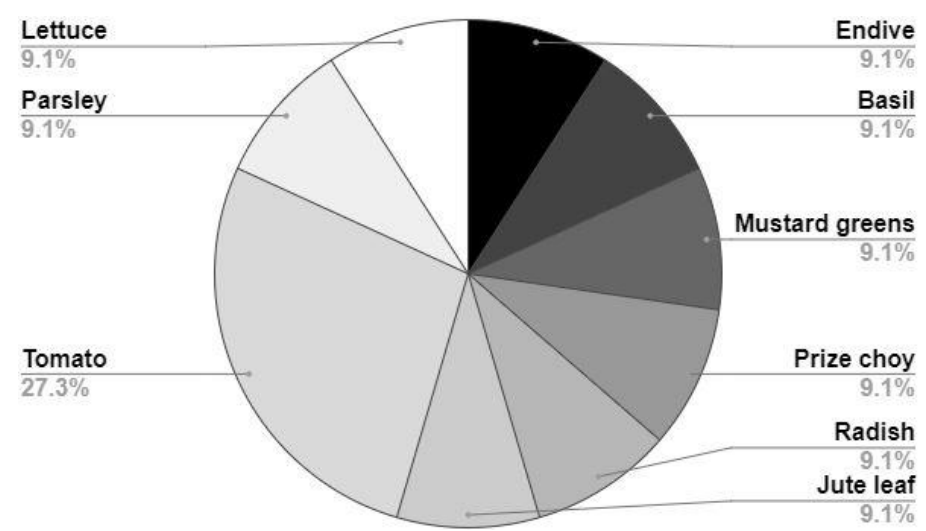

Figure 4: Types and frequency of appearance of vegetable cultures used for tests and validation for CEA systems described in the selected studies.

At the end of the sample review, it was possible to generate information to develop a small CEA system for research purposes. These are a list and analysis of models of sensors and actuators; mechanical and constructive aspects of the chambers; most popular quantities for data acquisition and systems control; and cultures that could be used in tests. Lastly, because of these outcomes, the application of the results refers to using them for building and validating a small growth chamber for research purposes.

After presenting and applying it, we would like to reiterate why the proposed systematic review methodology is better suited for electrical engineering than the already established ones.

First, traditional SLR protocols originate from EBM. In health sciences research, experimental and clinical practices, which are the analysis goals in SLRs, demand much more resources and preparation than in engineering $[1,6,7,14]$. As a result, the task of conducting randomized, double-blind clinical trials cannot be equated with most engineering experiments [6, 7]. It makes it necessary to have more intricate tools for extracting information and analyzing SLRs results in the health-related fields [7]. Also, there are several details to be considered for these secondary studies. Examples are the demographics of participants and blinding strategies, which are particulars of in vivo experiments - not usual in electrical engineering. It makes the SLR protocol larger, stricter, and more complex for the health sciences [6-8]. In Kitchenham (2004) [6], Kitchenham and Charters (2007) [7], and Dyba et al. (2005) [14], aspects regarding differences between conducting SLRs in engineering and health sciences are discussed in more depth.

Simultaneously, although the methodology employed by software engineering and its related fields can be extended to a large degree to another engineering, it focuses on the development and 
maintenance of software [9], as attested in others studies [6-9, 14, 16-19]. Seemingly, it was the right approach to introduce the SLR practice to the field. By conducting a quick search in the IEEE Xplore library, employing "systematic review" as the search string, there are 1156 results, with 99\% (1145 results) after 2004 - the inauguration of EBSE [6]. The publication topics are dominated by software engineering and computer science, with only 115 studies being healthcarerelated. Furthermore, software engineering and computer science deal with several aspects unrelated to electrical engineering. This means that, when focusing on software, important topics for electrical engineering, such as the development, evaluation, and characterization of equipment in different environmental conditions, are not covered in the procedures of a SLR for software engineering.

Introducing the practice of performing systematic literature reviews in electrical engineering, as we intend with this paper, may have an effect like what occurred in other areas, opening a niche of secondary studies. It would bring scientists closer to evidence-based decision making, rather than those based on market or industry trends or pressures [14].

\section{CONCLUSION}

This paper proposes a methodology for performing SLRs in electrical engineering, an area that lacks them. In general, the goal of systematic reviews is to standardize a protocol for conducting literature reviews that facilitates obtaining more objective, concrete, and useful results, as well as achieving easier reproducibility. By using it, researchers avoid or minimize problems like search, selection, and publication bias.

We applied the proposed methodology to run a case study on sensors, transducers, and actuators in small CEA systems for research purposes in the last ten years, obtaining a list of such elements and examples of cultures used for testing growth chambers. More importantly, the steps and the case study we described can be used as an easy-to-understand guide for electrical engineers to perform SLRs.

Despite the advances achieved, it is worth noting that the proposed methodology is not intended to replace review practices already established in the field of engineering. This is because in electrical engineering, the way of acquiring and treating evidence, in general, differs greatly from that found in the health sciences, and even in software engineering. However, when facing challenges with better-defined research parameters and a specific question regarding the research interest, the proposed protocol can become a powerful tool for the acquisition, summary and understanding of evidence already produced, strengthening the evidence-based decision-making process.

Finally, the developed protocol can also be a great ally in initiating researchers in review methodologies and in the dissemination of good research practices. This is because it presents the search process for references in a well-structured and objective way, contributing to the understanding of aspects related to the focus and specificity of research topics, selection of sources and studies, extraction of information and presentation of results.

\section{ACKNOWLEDGEMENTS}

This study was financed in part by the Coordenação de Aperfeiçoamento de Pessoal de Nível Superior - Brasil (CAPES) - Finance Code 001, and also by the Coordenação de Pesquisa (COPES) of the Federal University of Sergipe.

\section{REFERENCES}

1. Biolchini J, Mian PG, Natali ACC, Travassos G. Tecnhical Report ES 679/05: Systematic review in software engineering. Rio de Janeiro (RJ): Systems Engineering and Computer Science Department COPPE/UFRJ; 2005 May. 30 p.

2. Robinson P, Lowe J. Literature reviews vs systematic reviews. Australian and New Zealand Journal of Public Health. 2015;39(2):103-103, doi: 10.1111/1753-6405.12393 
3. Wright RW, Brand RA, Dunn W, Spindler KP. How to write a systematic review. Clinical Orthopaedics and Related Research (1976-2007). 2007 Feb;455:23-29, doi:10.1097/BLO.0b013e31802c9098

4. Ministério da Saúde, Secretaria de Ciência, Tecnologia e Insumos Estratégicos, Departamento de Ciência e Tecnologia (BR). Diretrizes metodológicas: elaboração de revisão sistemática e metanálise de ensaios clínicos randomizados. 1. ed. Brasília (DF): Editora do Ministério da Saúde; 2012. 92 p.

5. Hochman B, Nahas FX, Oliveira Filho RS, Ferreira LM. Research designs. Acta Cirúrgica Brasileira. 2005;20(Supl 2):2-9, doi: 10.1590/S0102-86502005000800002

6. Kitchenham B. Keele University Technical Report TR/SE-0401: Procedures for performing systematic reviews. Keele (UK): Software Engineering Group, Department of Computer Science, Keele University (UK); 2004 July. 33p.

7. Kitchenham B, Charters S. EBSE Technical Repor EBSE-2007-01: Guidelines for performing systematic literature reviews in software engineering. Ver. 2.3. Keele (UK): Software Engineering Group - Keele University (UK), Department of Computer Science - University of Durham (UK); 2007 July. 65 p.

8. Kitchenham BA, Budgen D, Brereton P. Evidence-based software engineering and systematic reviews. Vol. 4. Boca Raton (FL): CRC Press; 2015.

9. Kitchenham B, Brereton OP, Budgen D, Turner M, Bailey J, Linkman S. Systematic literature reviews in software engineering - a systematic literature review. Information and Software Technology. 2009;51(1):7-15, doi: 10.1016/j.infsof.2008.09.009

10. Kiran GR, editor. Proceedings of the 3rd MEC International Conference on Big Data and Smart City (ICBDSC); 2016 Mar 15-16; Muscat, Oman. Red Hook: IEEE; c2016. 381 p., doi: 10.1109/ICBDSC.2016.7460386

11. Shamshiri RR, Kalantari F, Ting K, Thorp KR, Hameed IA, Weltzien C, Ahmad D, Shad ZM. Advances in greenhouse automation and controlled environment agriculture: a transition to plant factories and urban agriculture. International Journal of Agricultural and Biological Engineering. 2018;11(1):1-22, doi: $10.25165 /$ j.ijabe.20181101.3210

12. Hee LM, editor. Proceedings of the Engineering Application of Artificial Intelligence Conference 2018 (EAAIC 2018); 2018 Dec 3-5; Sabah, Malaysia. Les Ulis (FR): EDP Sciences; c2019. 318 p., doi:10.1051/matecconf/201925502006

13. Krizek, DT. Guidelines for measuring and reporting environmental conditions in controlledenvironment studies. Physiologia Plantarum. 1982;56(3):231-235, doi: 10.1111/j.13993054.1982.tb00331.x

14. Dyba T, Kitchenham BA, Jorgensen M. Evidence-based software engineering for practioners. IEEE Software. 2005;22(1):58-65, doi: 10.1109/MS.2005.6

15. Bilof RS, editor. Proceedings of the 2019 7th International Engineering, Sciences and Technology Conference (IESTEC 2019); 2019 Oct 9-11; Panama City, Panama. New Jersey: IEEE Computer Society Conference Publishing Services (CPS); c2019. 706 p., doi:10.1109/IESTEC46403.2019.00-62

16. Martini A, Wimmer M, Skavhaug A, editors. Proceedings of the 46th Euromicro Conference on Software Engineering and Advanced Applications (SEAA 2020); Aug 26-28; Portoroz, Slovenia. New Jersey: IEEE Computer Society Conference Publishing Services (CPS); c2020. 600 p, doi: 10.1109/SEAA51224.2020.00077

17. Jorgensen M, Shepperd M. A systematic review of software development cost estimation studies. IEEE Transactions on Software Engineering. 2007;33(1):33-53, doi: 10.1109/TSE.2007.256943

18. Paul S, Verma JK, editors. Proceedings of the 2020 International Conference on Computational Performance Evaluation (ComPE); 2020 Jul 2-4; Sillong, India. Red Hook: IEEE; c2020. 868 p., doi: 10.1109/ComPE49325.2020.9200103

19. Dyba T, Dingsoyr T. Empirical studies of agile software development: a systematic review. Information and Software Technology. 2008;50(9-10):833-859, doi: 10.1016/j.infsof.2008.01.006 